\title{
Correction to: Heat Flux for a Relativistic Dilute Bidimensional Gas
}

\author{
A. L. García-Perciante ${ }^{1} \cdot$ A. R. Méndez ${ }^{1}$. \\ E. Escobar-Aguilar ${ }^{1,2}$
}

Published online: 9 August 2018

๑) Springer Science+Business Media, LLC, part of Springer Nature 2018

\section{Correction to: J Stat Phys (2017) 167:123-134 https://doi.org/10.1007/s10955-017-1742-x}

The original version of this article unfortunately contained a mistake.

Equation (4) should read $h^{\mu \nu}=\eta^{\mu v}-\frac{1}{c^{2}} \mathscr{U}^{\mu} \mathscr{U}^{v}$, the sign being minus due to the +-signature employed. The expression used in the rest of the manuscript is the correct one, and only Eq. (4) needs to be modified. Also, the right hand side of Eq. (24) should be multiplied by -1 in order to be consistent with Eq. (16). This leads to the relation $b_{1}=-a_{1} / g(z)$ (from Eqs. (23) and (24)) and thus to a missing minus sign in Eq. (38). The coefficient $L_{n}$ is then given by

$$
L_{n}=-\frac{30 m c^{3}}{d} \frac{z^{5}(2+3 z(2+z))^{2}}{\left(3 z^{2}+3 z+1\right)(1+z)} \frac{\exp \left(-\frac{2}{z}\right)}{I(z)}
$$

and the value plotted in Fig. 1 corresponds to its absolute value $\left|L_{n}\right| / L_{T_{N R}}$.

The original article can be found online at https://doi.org/10.1007/s10955-017-1742-x.

$凶$ A. L. García-Perciante

algarcia@correo.cua.uam.mx

A. R. Méndez

amendez@correo.cua.uam.mx

1 Departamento de Matemáticas Aplicadas y Sistemas, Universidad Autónoma Metropolitana - Cuajimapla, Av. Vasco de Quiroga 4871, 05348 Ciudad de México, México

2 Present Address: Departamento de Física, Universidad Autónoma Metropolitana - Iztapalapa, Av. San Rafael Atlixco 186, 09340 Ciudad de México, México 